\title{
Evaluation of Two Rapid Diagnostic Tests for Rabies Diagnosis under Field and Laboratory Conditions in Nigeria
}

\author{
Mshelbwala $\mathrm{PP}^{1^{*}}$, Abdullahi SU1 ${ }^{1}$, Maikai $\mathrm{BV}^{2}$, Onyiche $\mathrm{ET}^{3}$ and Ogunkoya $\mathrm{AB}^{1}$ \\ ${ }^{1}$ Department of Veterinary Medicine, Ahmadu Bello University, Zaria, Nigeria \\ ${ }^{2}$ Department of Veterinary Public Health and Preventive Medicine, Ahmadu Bello University, Zaria, Nigeria \\ ${ }^{3}$ Department of Veterinary Parasitology, University of Ibadan, Nigeria
}

*Corresponding author: Mshelbwala PP, Department of Veterinary Medicine, Ahmadu Bello University, Zaria, Nigeria, Tel: +2348035745529; E-mail: philbwala@yahoo.com

Received date: 06 December 2014; Accepted date: 11 February 2015; Published date: 16 February 2015

Copyright: (c) 2015 Mshelbwala PP, et al. This is an open-access article distributed under the terms of the Creative Commons Attribution License, which permits unrestricted use, distribution, and reproduction in any medium, provided the original author and source are credited.

\begin{abstract}
The study was carried out to evaluate two rapid diagnostic rabies diagnostic tests in South Eastern Nigeria. One hundred samples each of saliva and brains were collected before and after slaughter from apparently healthy dogs brought for slaughter. The saliva was subjected to rapid immune chromatographic test while the brain tissues were subjected Direct Fluorescent Antibody Test (DFAT) and direct Rapid Immunohistochemistry Test (dRIT). Five (5\%) tested positive for rabies antigen with the use of the three tests. Result obtained from this study have shown total agreement in the results of the entire tests used. This study therefore recommends the use of RICT and dRIT for rabies virus screening under field and laboratory conditions.
\end{abstract}

Keywords: Rabies; Nigeria; Diagnosis; DRIT; DFA; RICT

\section{Introduction}

Worldwide, rabies is among the most feared of all diseases as it has resulted in the loss of about 55,000 persons yearly according to the World Health Organization (WHO) [1]. Global Alliance for Rabies Control (GARC) report that the death is over 70,000 with 10 million treated for bites from potentially infected dogs. Majority of the cases occur in Africa and Asia although, the true incidence in Asia and Africa is likely to be higher than is reported as most cases are not reported [2]. In Nigeria, rabies is highly neglected due to poor surveillance and reporting systems, absence of diagnostic facilities, poor vaccination, and irresponsible dog ownership among others [3]. Rabies was first reported in 1912 in humans and 1925 in dogs in Nigeria [4]. Since the first report many workers have continued to report it in different parts of the country, signifying its endemicity [5-11]. Dog bites from owned dogs have resulted in the loss of lives in plateau state, North Central Nigeria [12]. Recently, eight lives due to dog bites were lost in South Eastern Nigeria, but no confirmatory diagnosis was made due to the absence of diagnostic facilities in the affected state and the culture of consuming dog in the state [13].

Nigeria has 36 states and the state capital. Out of these states, only two have facilities for rabies diagnosis, namely National Veterinary Research Institute, Vom Plateau State and Ahmadu Bello University, Zaria, Kaduna State and these states happen to be in the Northern part of the country.

Recently, a ten years retrospective study of dog bite cases reported to Zonal Veterinary Clinic, Umuahia, Abia state, South Eastern Nigeria by Mshelbwala, et al. [11] revealed that all the suspected cases of rabies were not sent for confirmatory diagnosis due to the distance to diagnostic facilities and the cost of transportation.
Some brain tissue samples received for diagnosis in Nigeria and other developing countries of Africa such as Niger, Chad, Cameroon, Ghana and Benin Republic get decompose due lack of storage facilities, inadequate transportation, lack of rapid test especially when exposure occur in rural areas [14], which may result in misdiagnosis and loss of human life. For rabies surveillance, control and eradication program to succeed, proper diagnosis is fundamental. The Direct Fluorescent Antibody Test (DFAT) is the gold-standard test approved by both WHO and OIE for rabies diagnosis [25]. Brain tissues from suspected rabid animals are collected and the test is performed on the samples. The test can only be performed at post-mortem. However, DFAT can only be performed in the laboratory requiring high technical skill and equipment [23]. Rapid and accurate laboratory diagnosis of rabies in humans and other animals is essential for timely administration of post-exposure prophylaxis [15]. If the animal is not rabid, prompt diagnosis may save a patient from unnecessary physical and psychological trauma, as well as financial burden [15].

Lembo et al. [16] had 100\% sensitivity and 100 specificity when he compared dRIT and DFAT in the diagnosis of rabies in Tanzania under field and laboratory conditions. To the best of our knowledge, this is the first study to compare the Rapid Immune Chromatographic Test (RICT) using saliva to that of the direct Rapid Immunohistochemistry Test (dRIT) and direct fluorescent antibody test (DFAT) using brain tissues. The dRIT test has also undergone extensive evaluation in other countries [17-19] and $100 \%$ correlation was found with DFAT. It is against this background that this study was designed with the aim of evaluating two rapid diagnostic tests suitable for use in Nigeria with the gold standard for rabies diagnosis. 


\section{Materials and Methods}

\section{Study area}

Located in the South-Eastern part of Nigeria, Abia State lies approximately latitudes $4^{\circ} 40^{\prime}$ and $6^{\circ} 14^{\prime}$ north of the equator and longitudes $7^{\circ} 10^{\prime}$ and $8^{\circ}$ east of the Greenwich meridian. The state shares common boundaries to the north with Ebonyi State; to the south and southwest with Rivers State; and to the east and southeast with Cross River and Akwa lbom States. To the west is Imo State, and to the northwest is Anambra State. The state covers an area of about $5,243.7$ sq. $\mathrm{km}$ which is approximately $5.8 \%$ of the total land area of Nigeria. The study was carried out in Umuahia north, Ekwuano, Osisioma and Isianlangwa north Local Government Areas of the State.

\section{Sampling method}

Convenient random sampling as described by Mike [20] was employed, and the most accessible units of the population were used. Live dogs were sampled prior to slaughter in Four (4) slaughtering points (Umuahia north, Ekwuano, Osisioma and Isianlangwa north Local Government Areas of the State (LGAs)) of the state.

A total of one hundred samples each of saliva and brains were collected during the period of study from the various slaughtering points within the study area. The saliva was obtained before slaughter while the brain was collected after slaughtering. Each slaughter point was visited once daily.

\section{Types of sample}

Saliva and brain samples were collected for the various assays: Saliva of dogs was sampled using the sterile swab stick which was inserted into the mouth of the dog before slaughter. Brains of dogs whose saliva was previously tested were extracted on the field using the method described by Kaplan and Koprowski [21]. The whole brain was removed, placed in a polytene bag, placed into an ice pack, it was then transported and stored in Thermo cool deep freezer at Animal World Veterinary Clinic, Umuahia, before being transported to National Veterinary Research Institute (NVRI), Vom where DFAT were carried out as described by Dean et al (1996), and Rabies laboratory of the Department of Veterinary Medicine, ABU, Zaria, where dRIT were carried out as described by CDC [22].

\section{Testing Procedure}

Rapid Immune Chromatographic Test (RICT): Swabs were then inserted into an assay buffer tube and stirred to ensure a good sample extraction. The immune chromatographic test cassette was removed and placed horizontally. Using a sterile dropper, three drops of the extracted sample was dipped into the sample hole in the cassette and the result was interpreted within 5-10 minutes according to manufacturer's instruction (Bio Note ${ }^{\circ}$ ).

Procedure for Direct Fluorescent Antibody Test (DFAT): Rabies direct fluorescent antibody assay DFAT (Monoclonal antibodyconjugate) reagents from Fujirebio Diagnostic Inc. Malvern, P.A 19355 were used and the working (reagent) dilution after titration was achieved at 1:40 in accordance with the manufacturers recommendations and as described by Flamand et al. [23]. A small fraction of the brain sample was smeared using wire loop on one part of a slide and then was air dried and fixed in cold acetone for one hour at $-20^{\circ} \mathrm{C}$. The slides were air dried and then the rabies conjugate was applied at 1:40 and incubated for 30 minutes at $37^{\circ} \mathrm{C}$ in a humid chamber after which excess conjugate was removed from the slides by rinsing it with 7.4 pH PBS solution about 3-5 minutes and was allowed to air dry. The cover slips were mounted with buffered Glycerol Mounting medium and the slides were examined using a fluorescence microscope within 2 hours after staining. When brilliant applegreen fluorescence colour or greenish yellow objects is exhibited against a black background the test slide is positive. If no specific applegreen fluorescence is exhibited the test slide is negative.

Direct Rapid Immunohistochemistry Test (DRIT): Following DFAT at NVRI, the same brain samples were transported to rabies laboratory of the Department of Veterinary Medicine, Ahmadu Bello University Zaria and stored at $-20^{\circ} \mathrm{C}$ in deep freezer. All the brain samples were subjected to DRIT as described by Lembo et al. [16].

- A routine touch impressions of each sampled brain tissue were made on labelled glass microscope slides (including the standard positive and negative controls).

- Slides were arranged in slides holder, air-dry for 5 minutes at room temperature.

- Slides were then immersed in $10 \%$ buffered formalin at room temperature for 10 minutes. Dish I

- Slides were then removed and dip-rinsed several (10X) times to wash off any excess fixative in wash buffer Tween phosphate buffer saline TPBS (PBS with $1 \%$ tween 80 ). Dish II.

- Slides were then immersed in $3 \%$ hydrogen peroxide $\left(\mathrm{H}_{2} \mathrm{O}_{2}\right)$ for 10 minutes. Dish III.

- Excess hydrogen peroxide was removed by dip-rinsing slides in TPBS, Dish IV. Slides were then Transferred into the next rinse Dish V containing TPBS (after dip-rinsing, excess buffer were shake off from slides edges using wipe papers surrounding the impression). A slide at a time was removed and wiped and were arranged on a wet paper towel on laboratory flat table top.

- Slides were then incubated in a humidity chamber (I used a moistened paper towel, on laboratory bench top covering with 96 well plastic cover) at room temperature after adding primary antibody-(biotinylated anti-rabies $\mathrm{mAb}$ ) for 10 minutes ( primary antibody usually 2-3 drops were added using plastic pipettes).

- After incubation with primary antibody, excess conjugate were shake off. Slides dip-rinsed in TPBS, Dish V (excess TPBS were shake off and blot buffer from slides edges surrounding the impression using wipe paper). This same wash buffer was used through step 10.

- Slides were placed back on the wetted paper towel then incubated with Streptavidin-peroxidase complex (2-3 drops were added) in humidity chamber as described in step 7 above at room temperature for 10 minutes. After incubation, slides were shakeoff.

- Slides were then dip-rinsed with TPBS, in Dish V (excess buffer shake off and blotted from slide edges surrounding the impression).

- Slides were then incubated with peroxidase substrate, aminoethylcarbizole (AEC)-the working dilution was prepared just prior to use. Enough of this substrate was added to the slide by drop to cover the impression in a humidity chamber (as in step 7 above) at room temperature for 10 minutes. After incubation, shake off excess substrate.

- Slides were then dip -rinsed in de-ionized/distilled water, Dish VI. 
Citation: Mshelbwala PP, Abdullahi SU, Maikai BV, Onyiche ET, Ogunkoya AB (2015) Evaluation of Two Rapid Diagnostic Tests for Rabies Diagnosis under Field and Laboratory Conditions in Nigeria. J Vaccines Vaccin 6: 272. doi:10.4172/2157-7560.1000272

Page 3 of 5

- Then counter stain with Gills Hematoxylin (diluted 1:2 with deionized/distilled water) for 2 minutes Dish VII.

- Immediately after the 2 minutes counter staining, the slides were dip-rinsed (10 times) with de-ionized/distilled water in Dish VIII. A second dip-rinse of slides with fresh deionized/distilled water (Dish IX) was made to ensure removal of excess stain.

- Slides were then transferred to fresh distilled water Dish X. Slides were mounted with cover slips using water-soluble mounting medium.

- Slides were viewed by light microscopy, using a $20 \mathrm{x}$ objective to scan the field, and a 40x objective for higher power magnification.

- Rabies viral antigen appeared as red coloured particles/inclusions under a bluish neuronal background indicated the samples was positive. A complete bluish background suggested that the sample was negative.

\section{Results}

A total of 100 (both saliva and brain samples from the same animal) samples were collected. From the saliva samples, $5(5 \%)$ tested positive for rabies antigen by the RICT (Figure 1). Result of the brain tissue samples also revealed that $5(5 \%)$ were positive for DRIT and DFAT (Figure 1). A total of 25 samples each of saliva and brain were collected from the selected four LGAs. Two samples were positive from Umuahia North, 2 from Isialangwa North, 1 from Ikwuano, while none of the samples obtained from Osisioma was positive (Table 1). Figure 2, shows the result of RICT test. Around 5 min after the diluted samples were placed in the device of the RICT kit, 5 tested positive for viral antigen, with the development of two colored lines. The intensities of the test lines varied between samples while 95 of the saliva samples tested negative for antigen, with the appearance of one colored line. The result of the DFAT shows apple-green viral inclusions bodies (Figure 2) while that of DRIT has a bluish particle in a neuron spreading along dendrites and axon (Figures 3 and 4).

\begin{tabular}{|l|l|l|}
\hline Location & No of samples & No Positive (\%) \\
\hline Umuahia North & 25 & $2(8)$ \\
\hline Ikwuano & 25 & $1(4)$ \\
\hline Isianlangwa North & 25 & $2(8)$ \\
\hline Osisioma & 25 & $0(0)$ \\
\hline Total & 100 & $5(5)$ \\
\hline
\end{tabular}

Table 1: Distribution of samples by location.

\section{Discussion}

Rabies is one of the oldest diseases infecting animals, including humans, and it continues to infect animals in spite of the availability of effective vaccines and antiserum to prevent the disease [15]. Result obtained from this study have shown total agreement in the results of the entire tests used, although previous works have been carried out to evaluate RICT with DFAT under laboratory condition using antemortem sample and DRIT with DFAT, using post mortem samples. However, in our study we compared two rapid tests with the gold standard test for rabies, under laboratory condition.

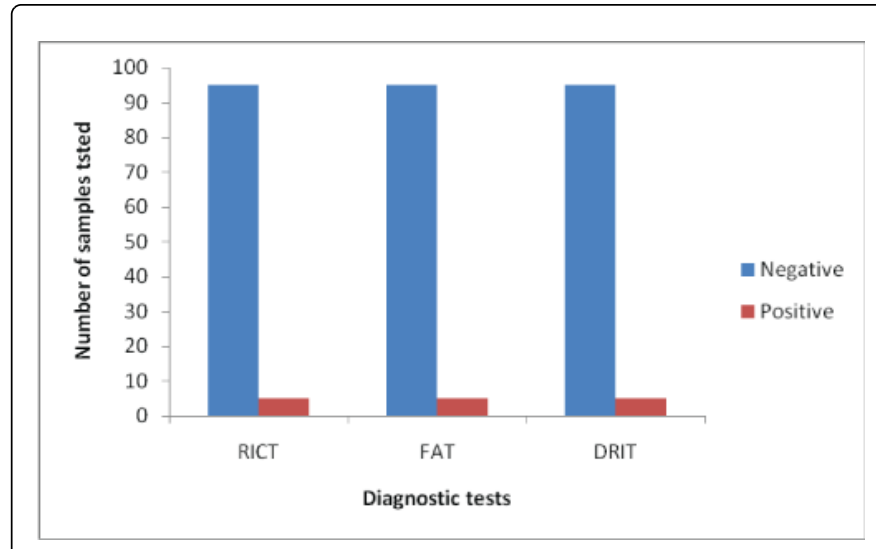

Figure 1: Results of the diagnostic Tests used.

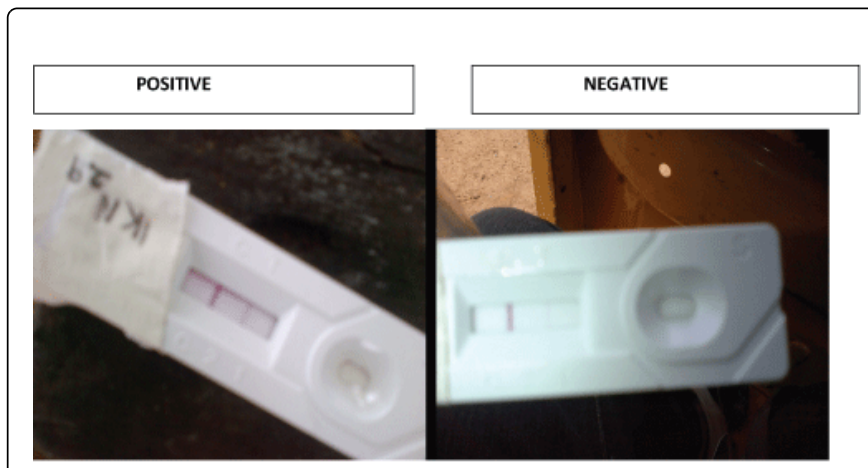

Figure 2: Result of RICT for Saliva sample, showing Positive and Negative results.

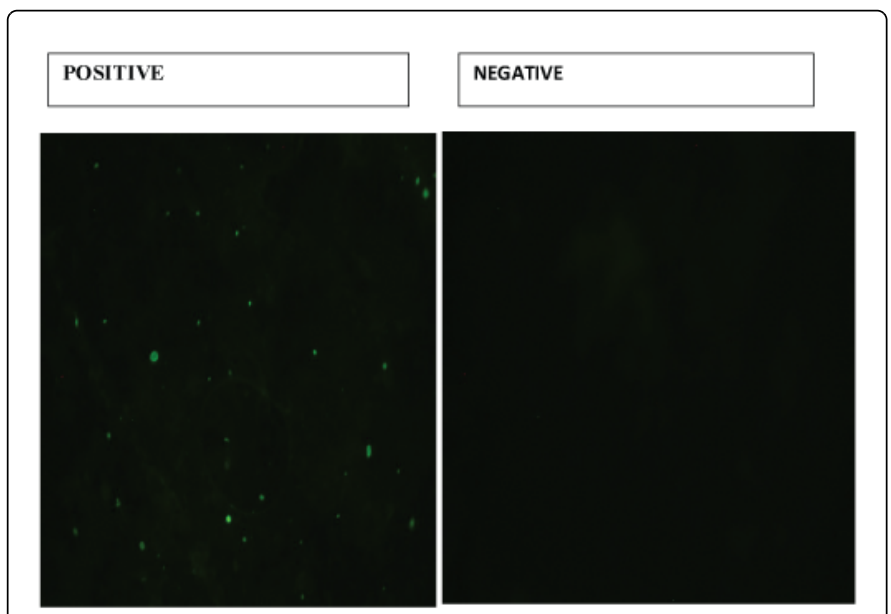

Figure 3: Result of FAT of the brain tissue.

The result obtained in this study revealed high sensitivity and specificity for rabies antigen using all the tests, as demonstrated in this study, however the result obtained here cannot be conclusive as there is possibility of false negative result with the use of RICT reported by other authors. The result slightly differs with the findings of Bokyu et al. [24] who obtained $100 \%$ sensitivity and $91.8 \%$ specificity in South 
Korea. Alexandre et al. obtained 100 specificity and $88 \%$ sensitivity. He also observed in their work that glycerol buffer used for storage affected the capillary flow and attributed it to false negative results. However in our studies, saliva was obtained fresh at the time of slaughter and brain was stored in refrigerator, this could be the reason for high sensitivity and specificity in our study.

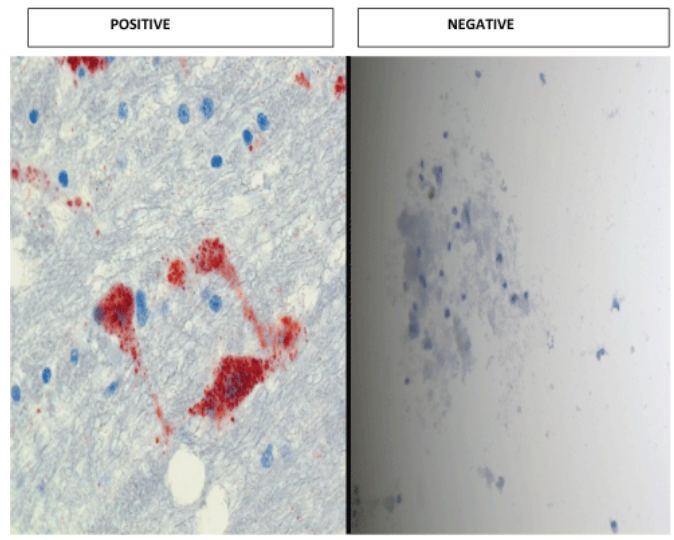

Figure 4: Result of DRIT for brain tissue.

The use of RICT and DRIT in diagnosis will reduce the number of human deaths due to rabies and financial loss incurred during PEP, because the results obtained is prompt and will determine if PEP is to be given or not. Both tests are simple to conduct under laboratory condition. They do not require the use of specialized equipment. DRIT can be performed on samples preserved under different conditions (Lembo et al. [15]). However, RICT, can be used on ante-mortem samples. This will help to prevent the unnecessary killing and beheading of dogs to collect the brain sample for confirmatory DFAT test. Dogs serve various purposes in Nigeria such as security, hunting, herding, in event such dogs are killed for rabies diagnosis and are found to be negative, the above listed purposes are defeated resulting in waste.

Additionally, the light microscope used in DRIT is cheaper compared to the costs of acquiring and maintaining a fluorescent microscope used in the DFAT test; it shows DRIT is less expensive. It is therefore more suitable for use in low income countries like Nigeria, especially in rural areas where rabies diagnosis is not available. We therefore, recommend the use of both tests for field diagnosis of rabies in Nigeria.

In conclusion, the rapid immunodiagnostic test kit is a useful method for rapid detection of rabies virus under various conditions. To prevent rabies effectively, rapid diagnosis in the field is necessary. The advantages of the RIDT kit over other testing protocols include rapidity, simplicity, minimal training needs for personnel, diversity of sample (including saliva) as opposed to DFAT which can only test brain tissue,. The RIDT kit can be used virtually anywhere like in the laboratories or in the field. The cost effectiveness of these diagnostic tests as well as the rapid reduction in time lag indicates that in the future, they will be a viable option in the diagnosis of rabies worldwide. However, it is advisable not to use one method alone but a combination of two to validate the outcome of the result, especially in cases of human exposure, due to possibility of false negative result and intermittent salivary shading of rabies virus. In the report of
Mshelbwala et al. [2] the saliva of the calf was negative with RIDT, however the brain was positive with RIDT and FAT.

\section{Acknowledgements}

The authors acknowledge Drs., Modupe Osinubi and Micheal Niezgoda of the Centers for Disease Prevention and Control, CDC, Atlanta who donated free DRIT kits used in this study, special thanks are to Mrs. Sarah who did the FAT and Drs. Ajoke and Garba Ahmed who assisted with DRIT. The first author (Dr. Philip Paul Mshelbwala) acknowledges the Federal Government of Nigeria for postgraduate scholarship he received part of which was used in this study.

\section{References}

1. World Health Organization (2013) WHO Expert Consultation on Rabies. Second report. World Health Organ Tech Rep Ser : 1-139, back cover.

2. Mshelbwala PP, Audu SW, Ogunkoya AB, Okaiyeto SO, James AA, et al. (2013) A case study of rabies in a six month old calf in zaria, Nigeria. J Exp Bio Agri Sci 1: 218-222.

3. Ogunkoya $\mathrm{AB}$ (2008) Review of rabies and problems of Rabies in Nigeria. Proceedings of the National conference/Work on rabies. Ahmadu Bello University, Zaria. Nigeria, 62-70.

4. Boulger LK, Hardy J (1960) Rabies in Nigeria. West Afr Med J 9: 223-234.

5. Umoh JU, Belino ED (1979) Rabies in Nigeria. A historical review. Int J Zoonoses 6: 41-48.

6. Fagbami AH, Anosa VO, Ezebiru EO (1981) Epidemiology of Rabies in Northern Nigeria. Trans Royal Soc Trop Med Hyg 75: 268-272.

7. Harry TO, Adeiga A, Anyiwo CE, Nasidi A (1984) Anti-rabies treatment of dog-bite victims in Lagos, Nigeria: trial of suckling mouse brain and fetal bovine kidney cell rabies vaccines. Vaccine 2: 257-260.

8. Ajayi BB1, Rabo JS, Baba SS (2006) Rabies in apparently healthy dogs: histological and immunohistochemical studies. Niger Postgrad Med J 13: 128-134.

9. Garba A, Oboegbulem SI, Elsa AT, Janaidu AU, Magaji AA, et al. (2008) A comparative rabies laboratory diagnosis: Peculiar features of samples from apparently healthy dogs in Nigeria. Sok J Vet Sci 7: 33-36.

10. Aliyu TB, De N, Yenda EN, Lynn M (2010) Prevalence of rabies virus antigens in apparently healthy dogs in Yola, Nigeria. Researcher 2: 4-10.

11. Mshelbwala PP, Ogunkoya AB, Maikai BV (2013) Detection of Rabies Antigen in the Saliva and Brains of Apparently Healthy Dogs Slaughtered for Human Consumption and Its Public Health Implications in Abia State, Nigeria. ISRN Veterinary Science.

12. Ogunkoya AB, Audu SW, Osinubi MOV, NwannenaVC, AbaTC, et al. (2012) Two Fatal cases of Rabies as a Result of bites from apparently healthy dogs Presented at World Rabies International WEBINAR. Office Internatironal des Epizooties 2012. Immediate notification report.

13. Ogunkoya AB (2010) Rabies in Nigeria: Problems and complications militating against its eradication and control. Paper presented at 2nd National Workshop on Rabies, IDR-Ahmadu Bello University, Zaria, Nigeria.

14. Helmick CG (1983) The epidemiology of human rabies postexposure prophylaxis, 1980-1981. JAMA 250: 1990-1996.

15. Lembo T, Niezgoda M, Velasco-Villa A, Cleaveland S, Ernest E, et al. (2006) Evaluation of a direct, rapid immunohistochemical test for rabies diagnosis. Emerg Infect Dis 12: 310-313.

16. Madhusudana SN, Subha S, Thankappan U, Ashwin YB (2012) Evaluation of a direct rapid immunohistochemical test (dRIT) for rapid diagnosis of rabies in animals and humans. Virol Sin 27: 299-302.

17. Dürr S, Naïssengar S, Mindekem R, Diguimbye C, Niezgoda M, et al. (2008) Rabies diagnosis for developing countries. PLoS Negl Trop Dis 2: e206.

18. Tao XY, Niezgoda M, Du JL, Li H, Wang XG, et al. (2008) [The primary application of direct rapid immunohistochemical test to rabies diagnosis 
Citation: Mshelbwala PP, Abdullahi SU, Maikai BV, Onyiche ET, Ogunkoya AB (2015) Evaluation of Two Rapid Diagnostic Tests for Rabies Diagnosis under Field and Laboratory Conditions in Nigeria. J Vaccines Vaccin 6: 272. doi:10.4172/2157-7560.1000272

Page 5 of 5

in China]. Zhonghua Shi Yan He Lin Chuang Bing Du Xue Za Zhi 22: 168-170.

19. http://snrmo.sagepub.com/view/encyclopedia-of-survey-surveymethods /n535

20. http://www.cdc.gov/ncidod/dvrd/rabies

21. Flamand A, Wiktor TJ, Koprowski H (1980) Use of hybridoma monoclonal antibodies in the detection of antigenic difference between rabies virus and rabies related virus proteins. The nucleocapsid protein. J Gen Virol 48: 105-109.
22. Kang B, Oh J, Lee C, Park BK, Park Y, et al. (2007) Evaluation of a rapid immunodiagnostic test kit for rabies virus. J Virol Methods 145: 30-36.

23. Servat A, Picard-Meyer E, Robardet E, Muzniece Z, Must K, et al. (2012) Evaluation of a Rapid Immunochromatographic Diagnostic Test for the detection of rabies from brain material of European mammals. Biologicals 40: 61-66.

24. World Health Organization (2013) WHO Expert Consultation on Rabies. Second report. World Health Organ Tech Rep Ser : 1-139, back cover. 